\title{
A NOVEL BAT ALGORITHM FOR SOLVING OPTIMAL POWER FLOW PROBLEM
}

\author{
Hardiansyah Hardiansyah ${ }^{1^{*}}$
}

Department of Electrical Engineering, Faculty of Engineering, University of Tanjungpura, Indonesia

\begin{tabular}{|c|c|}
\hline ARTICLE INFO & Abstract: \\
\hline $\begin{array}{l}\text { Article history: } \\
\text { Received: } 21.05 .2019 . \\
\text { Received in revised form: } 24.09 .2019 . \\
\text { Accepted: } 24.09 .2019 .\end{array}$ & \multirow{7}{*}{$\begin{array}{l}\text { This paper presents an application of a novel bat algorithm } \\
\text { (NBA) for solving optimal power flow (OPF) problems in power } \\
\text { systems. The proposed algorithm combines a bat habitat selection } \\
\text { and their self-adaptive compensation for the Doppler effects in } \\
\text { echoes into the basic bat algorithm (BA). The selection of the bat } \\
\text { habitat is modeled as a selection between their quantum behavior } \\
\text { and mechanical behavior. The objective of this paper is to } \\
\text { minimize the total generation costs by considering equality and } \\
\text { inequality constraints. To validate the proposed algorithm, the } \\
\text { standard IEEE } 30-\text { bus and 57-bus test systems are applied. The } \\
\text { results show that the proposed technique provides a better } \\
\text { solution than other heuristic techniques reported in the literature. }\end{array}$} \\
\hline Keywords: & \\
\hline Novel bat algorithm & \\
\hline Optimization & \\
\hline Optimal power flow & \\
\hline Power systems & \\
\hline DOI: https://doi.org/10.30765/er.1465 & \\
\hline
\end{tabular}

\section{Introduction}

A modern power system consists of a set of connections in which the energy can be transmitted from generators to a load. In an interconnected power system, the objective is to find the real and reactive power scheduling of each power plant in such a way as to minimize the operating cost. This means that the generator's real and reactive power is allowed to vary within certain limits so that it can meet the demand with a minimum fuel cost. This is called the optimal power flow. The optimal power flow (OPF) problem has become an essential for operation, planning and control of the power systems. It was proposed first time in 1968 by Dommel and Tinney [1]. The main goal of the OPF problem is to optimize a selected objective function such as fuel cost, power loss, etc. In solving the OPF problem, objective function is optimized by adjusting system control variable while satisfying the equality and inequality constraints. The equality constraints normally power the flow equations and inequality constraints present limits on control variables and limits of the power system dependant variables. Many conventional techniques such as gradient-based method, Newton method, linear programming, and quadratic programming have been employed for the solution of the OPF problem [2-4]. But most of these methods cannot find a global optimal solution in the OPF problems which have nonlinear constraints and objective functions. Recently, numerous heuristic algorithms have been developed and implemented to successfully generate the OPF solution such as tabu search (TS) [5], genetic algorithm (GA) [6-8], evolutionary programming (EP) [9], artificial bee colony (ABC) algorithm [10-12], differential evolution (DE) [13-16], teaching learning-based optimization (TLBO) [17-19], biogeography-based optimization (BBO) [20-22], particle swarm optimization (PSO) [23-25], and gravitational search algorithm (GSA) [26].

Nowadays, to find out the optimized solution of the complex problem evolutionary algorithms algorithmic models are used. Meta-heuristic algorithms are based on natural phenomenon and are suitable for the complex optimization problems. These algorithms solve the optimization problem according to population and keep on searching and evaluating for a number of times until an optimized result is obtained. New algorithms developed are either nature inspired or are dependent on the behavior of animals. Apart from the development of new optimization algorithms, many new variations of a particular algorithm are being continuously developed. One such instance is the bat algorithm (BA), which was originally proposed in [27]-

\footnotetext{
* Corresponding author.

E-mail address: hardiansyah@ee.untan.ac.id
} 
[29] and has seen a variety of modifications being incorporated to find which among them proves to be the most optimal. In other words, variations of the same algorithm are developed to determine the best solution of a particular problem in a particular field of study. A new variation of the novel bat algorithm (NBA) has been discussed in [31,32] and applied for economic dispatch problem.

In this paper, a newly developed heuristic optimization called NBA method is proposed to solve the OPF problem which is formulated as a nonlinear optimization problem with the equality and inequality constraints in the power system. The performance of the proposed approach has been demonstrated on the standard IEEE 30-bus and IEEE 57-bus test systems. The simulation results obtained indicate that the proposed method gives better results than other heuristic techniques.

\section{Problem formulation}

The essential goal of the OPF is to optimally adjust the power system control variables in terms of a certain objective function while to satisfy various equality and inequality constraints at the same time. Generally, the OPF problem can be mathematically written as follows:

$$
\text { Min } J(x, u)
$$

Subject to

$$
\begin{aligned}
& g(x, u)=0 \\
& h(x, u) \leq 0
\end{aligned}
$$

where $J$ is objective function to be minimized, $g$ is the equality constraints represent typical load flow equations, $h$ is the inequality constraints represent the system operating constraints, $x$ is the vector of dependent variables or state vector consisting of:

(1) Active power of generators at slack bus $P_{G 1}$.

(2) Load bus voltage $V_{L}$.

(3) Generator reactive power output $Q_{G}$.

(4) Transmission line loading (line flow) $S_{l}$.

Hence, $x$ can be expressed as:

$$
x^{T}=\left[P_{G 1}, V_{L 1} \cdots V_{L \mathrm{NL}}, Q_{G 1} \cdots Q_{G \mathrm{NG}}, S_{l 1} \cdots S_{l \mathrm{ln}}\right]
$$

where NG, NL, and $\mathrm{nl}$ are the number of generators, number of load buses, and number of transmission lines, respectively. $u$ is the vector of the independent variables or control variables consisting of:

(1) Generator voltage $V_{G}$ at $\mathrm{PV}$ bus.

(2) Generator real power output $P_{G}$ at PV buses except at the slack bus $P_{G 1}$.

(3) Transformer tap setting $T$.

(4) Shunt VAR compensation (or reactive power of switchable VAR sources) $Q_{c}$.

Hence, $u$ can be expressed as:

$$
u^{T}=\left[P_{G 2} \cdots P_{G \mathrm{NG}}, V_{G 1} \cdots V_{G \mathrm{NG}}, Q_{c 1} \cdots Q_{c \mathrm{Nc}}, T_{1} \cdots T_{\mathrm{NT}}\right]
$$

where NT and NC are the number of the regulating transformer and VAR compensators, respectively.

\subsection{Objective Function}

The objective function for the OPF reflects the cost associated with generating the power system. The objective function for the whole power system can then be written as the sum of the fuel cost model for each generator:

$$
J=\sum_{i=1}^{\mathrm{NG}} F_{i}
$$


where $F_{i}$ indicate the fuel cost of the $i$-th generator.

The generator cost characteristics are defined as quadratic cost functions of the generator power output and the objective function selected.

$$
F_{i}=a_{i}+b_{i} P_{G i}+c_{i} P_{G i}^{2}
$$

where $a_{i}, b_{i}$, and $c_{i}$ are the cost coefficient of the $i$-th generator, $P_{G i}$ is the power generated by the $i$-th unit and NG is the number of generators.

\subsection{The Equality Constraints}

These constraints are specific load flow equations which can be described as follows:

$$
\begin{gathered}
P_{G i}-P_{D i}-V_{i} \sum_{j=1}^{\mathrm{NB}} V_{j}\left[G_{i j} \cos \left(\delta_{i}-\delta_{j}\right)+B_{i j} \sin \left(\delta_{i}-\delta_{j}\right)\right]=0 \\
Q_{G i}-Q_{D i}-V_{i} \sum_{j=1}^{\mathrm{NB}} V_{j}\left[G_{i j} \sin \left(\delta_{i}-\delta_{j}\right)-B_{i j} \cos \left(\delta_{i}-\delta_{j}\right)\right]=0
\end{gathered}
$$

where, $i=1, \ldots, \mathrm{NB}, \mathrm{NB}$ is the number of buses; $P_{G}$ is the active power generated, $Q_{G}$ is the reactive power generated, $P_{D}$ is the load active power, $Q_{D}$ is the load reactive power, $G_{i j}$ and $B_{i j}$ respectively indicate the real part and imaginary part of the $i j$-th element of the node admittance matrix.

\subsection{The Inequality Constraints}

These constraints reflect the system operating limits as follows [23]:

1. Generator constraints: generator voltages, real power outputs, and reactive power outputs are restricted by their lower and upper limits as follows:

$$
\begin{aligned}
V_{G i}^{\min } & \leq V_{G i} \leq V_{G i}^{\max }, i=1, \ldots, \mathrm{NG} \\
P_{G i}^{\min } & \leq P_{G i} \leq P_{G i}^{\max }, i=1, \ldots, \mathrm{NG} \\
Q_{G i}^{\min } & \leq Q_{G i} \leq Q_{G i}^{\max }, i=1, \ldots, \mathrm{NG}
\end{aligned}
$$

2. The transformer constraints: transformer tap settings are bounded as follows:

$$
T_{i}^{\min } \leq T_{i} \leq T_{i}^{\max }, i=1, \ldots, \mathrm{NT}
$$

3. Shunt VAR constraints: shunt VAR compensations are qualified by their limits as follows:

$$
Q_{c i}^{\min } \leq Q_{c i} \leq Q_{c i}^{\max }, i=1, \ldots, \mathrm{NC}
$$

4. Security constraints: these include the constraints of voltages at load busses and transmission line loadings as follows:

$$
V_{L i}^{\min } \leq V_{L i} \leq V_{L i}^{\max }, i=1, \ldots, \mathrm{NL}
$$




$$
S_{l i} \leq S_{l i}^{\max }, i=1, \ldots, \mathrm{nl}
$$

\section{Overview of the bat algorithm (BA)}

The Bat algorithm is a meta-heuristic approach that is based on echolocation behavior of bats. A bat has the capability to find its prey in a complete darkness. This was developed by Xin-She Yang in 2010 [27]. The algorithm mimics the echolocation behavior most prominent in bats. Bats fly randomly in the air or in the process of searching for prey by using echolocation to catch food and to avoid obstacles. This echolocation characteristic is copied in the virtual Bat algorithm with the following assumptions [27, 28]:

(1) All bats use echolocation mechanism to sense distance and they could distinguish between the prey and obstacles.

(2) Each bat randomly with a velocity $v_{i}$ at position $x_{i}$ with a fixed frequency $f_{\min }$, varying wavelength $\lambda$ and loudness $A_{0}$ searches for prey. They adjust the wavelength (or frequency) of their emitted pulses and adjust the rate of pulse emission $r \in[0,1]$, depending on the distance of the prey.

(3) Although loudness may vary in many ways, it is assumed that the loudness varies from a large (positive) $A_{0}$ to a minimum constant value $A_{\min }$.

\subsection{Initialization of the Bat Algorithm}

Initialization population of bats generated randomly in between the lower and upper boundary can be achieved by the following equation [29].

$$
x_{i j}=x_{\min j}+\operatorname{rand}(0,1)\left(x_{\max j}-x_{\min j}\right)
$$

where $i=1,2, \cdots, n ; j=1,2, \cdots, d ; x_{\min j}$ and $x_{\max j}$ are lower and upper boundaries for dimension $j$, respectively.

\subsection{Movement of Virtual Bats}

In the BA, the step size of the solution is controlled with the frequency factor. It is generated randomly between the minimum and maximum frequency $\left[f_{\min }, f_{\max }\right]$. The velocity of a solution is proportional to frequency, and a new solution depends on its new velocity, and it is represented as [29]:

$$
\begin{gathered}
f_{i}=f_{\min }+\left(f_{\max }-f_{\min }\right) \beta \\
v_{i}^{t}=v_{i}^{t-1}+\left(x_{i}^{t}-x_{\text {best }}\right) f_{i} \\
x_{i}^{t}=x_{i}^{t-1}+v_{i}^{t}
\end{gathered}
$$

where $\beta \in[0,1]$ indicates randomly generated number, $x_{\text {best }}$ represent current global best solutions. For local search part of algorithm (exploitation) one solution is selected among the selected best solutions and random walk is applied.

$$
x_{\text {new }}=x_{\text {old }}+\varepsilon A^{t}
$$

where $\varepsilon \in[-1,1]$ is a random number, while $A=<A_{i}^{t}>$ is the average loudness of all the bats at time step $t$.

\subsection{Loudness and pulse emission}

As the iteration proceed, the loudness and pulse emission have to updated because when batsget closer to their prey then they hear loudness. It usually decreases and pulse emission rate also increases, the updating equation for loudness and pulse emission is given by [29]: 


$$
\begin{gathered}
A_{i}^{t+1}=\alpha A_{i}^{t} \\
r_{i}^{t+1}=r_{i}^{0}[1-\exp (-\gamma t)]
\end{gathered}
$$

where $\alpha$ and $\gamma$ are constants. Actually, $\alpha$ is similar to the cooling factor of a cooling schedule in the simulated annealing. For simplicity, we set $\alpha=\gamma=0.9$ in our simulations.

The basic step of BA can be summarized as pseudo code shown in Table 1 [29].

Table 1. Pseudo code of the BA.

Bat algorithm
Objective function $f(x), x=\left(x_{1}, \cdots, x_{d}\right)^{T}$
Define pulse frequency $f_{i}$ at $x_{i}$
Initialization of the bat population $x_{i}(i=1,2, \ldots, n)$ and velocity $v_{i}$
Initialization of pulse rates $r_{i}$ and the loudness $A_{i}$
while $(t<$ Max number of iterations)
Generate new solutions by adjusting frequency
and updating velocities and locations/solutions (equations $(17)$ to (19))
if $\left(\right.$ rand $\left.>r_{i}\right)$
Select a solution among the best solutions
Generate a local solution around the current best solution
end if
Generate a new solution by flying randomly
if $\left(\right.$ rand $<A_{i} \& \&$ f $\left.\left(x_{i}\right)<f\left(x_{\text {best }}\right)\right)$
Accept the new solutions
Increase $r_{i}$ and reduce $A_{i}$
end if
Ranks bats and finds current best $x_{\text {best }}$
end while

\section{The novel bat algorithm (NBA)}

In the BA, the Doppler Effect and the idea of foraging of bats was not taken into consideration. In the original BA, each virtual bat is represented by its velocity and position, searches its prey in a $D$-dimensional space, and its trajectory is obtained. Also, according to the BA, it is considered that the virtual bats would forage only in one habitat. However, in fact, this is not always the case. In the NBA [30], the Doppler Effect has been included in the algorithm. Each virtual bat in the proposed algorithm can also adaptively compensate for the Doppler effects in echoes.

Meanwhile, the virtual bats are considered to have diverse foraging habitats in the NBA. Due to the mechanical behavior of the virtual bats considered in the BA, they search for their food only in one habitat. However, the bats in the NBA can search for food in diverse habitats. In summary, the NBA consists of the following idealized rules for mathematical formulation purposes.

(1) All bats can move around in different habitats.

(2) All bats can offset for the Doppler Effect in echoes. They can adapt and adjust their compensation rate depending upon the proximity of their targets.

\subsection{Quantum behavior of bats}

It is assumed that the bats will behave in such a manner that as soon as one bat finds food in the habitat, other bats would immediately start feeding from them. During the searching process, according to certain probability of mutation $p_{m}$, some bats will be mutated with the quantum behavior [30]; these bats are updated with the following formulas: 


$$
x_{i j}^{t+1}=\left\{\begin{array}{l}
g_{j}^{t}+\theta * \mid \text { mean }_{j}^{t}-x_{i j}^{t} \mid * \ln \left(\frac{1}{u_{i j}}\right) ; \text { if } \text { rand }_{j}(0,1)<0.5 \\
g_{j}^{t}-\theta * \mid \text { mean }_{j}^{t}-x_{i j}^{t} \mid * \ln \left(\frac{1}{u_{i j}}\right) ; \text { otherwise }
\end{array}\right.
$$

\subsection{Mechanical behavior of bats}

If the speed of sound in the air is $340 \mathrm{~m} / \mathrm{s}$, then this speed cannot be exceeded by the bats. Also, the Doppler Effect is compensated by the bats and this compensation rate has been mathematically represented as CR. It varies among different bats. A value $\xi$ is considered as the smallest constant in the computer to avoid the possibility of division by zero. The value of $\mathrm{CR} \in[0,1]$ and the inertia weight $w \in[0,1]$.

Here, if bats do not compensate for the Doppler Effect at all, then CR is assigned as 0, if they compensate fully, CR is assigned as 1 . Now, the following mathematical equations explain the description [30]:

$$
\begin{gathered}
f_{i j}=f_{\min }+\left(f_{\max }-f_{\min }\right) * \operatorname{rand}(0,1) \\
f_{i j}=\frac{c+v_{i j}^{t}}{c+g_{j}^{t}} * f_{i j} *\left(1+C R_{i} * \frac{g_{j}^{t}-x_{i j}^{t}}{\left|g_{j}^{t}-x_{i j}^{t}\right|+\xi}\right) \\
v_{i j}^{t+1}=w * v_{i j}^{t}+\left(g_{j}^{t}-x_{i j}^{t}\right) * f_{i j} \\
x_{i j}^{t+1}=x_{i j}^{t}+v_{i j}^{t}
\end{gathered}
$$

\subsection{Local Search}

When bats get closer to their prey, it is logical to assume that they would decrease their loudness and increase the pulse emission rate. Apart from whatever loudness they use, the factor of loudness in the surrounding environment also needs to be considered. This means the mathematical equations are developed as follows for the new bat positions in the local area and are given by the below-mentioned equations, where rand $n\left(0, \sigma^{2}\right)$ is a Gaussian distribution with the mean 0 and $\sigma^{2}$ as standard deviation [30]. At time step $t$, the mean loudness of all bats is $A_{\text {mean }}^{t}$.

$$
\begin{gathered}
\text { If }\left(\operatorname{rand}(0,1)>r_{i}\right) \\
x_{i j}^{t+1}=g_{j}^{t} *\left(1+\operatorname{rand} n\left(0, \sigma^{2}\right)\right) \\
\sigma^{2}=\left|A_{i}^{t}-A_{\text {mean }}^{t}\right|+\xi
\end{gathered}
$$

The pseudo code of the NBA is presented in Table 2 [30].

Table 2. Pseudo code of NBA.

\begin{tabular}{c} 
Novel bat algorithm \\
\hline Objective function $f(x), x=\left(x_{1}, \cdots, x_{d}\right)^{T}$ \\
Initialization of the bat population $x_{i}(i=1,2, \ldots, n)$ and \\
$v_{i}$ \\
Define pulse frequency $f_{i}$ at $x_{i}$ \\
Initialization of pulse rates $r_{i}$ and the loudness $A_{i}$ \\
$t=0 ;$
\end{tabular}




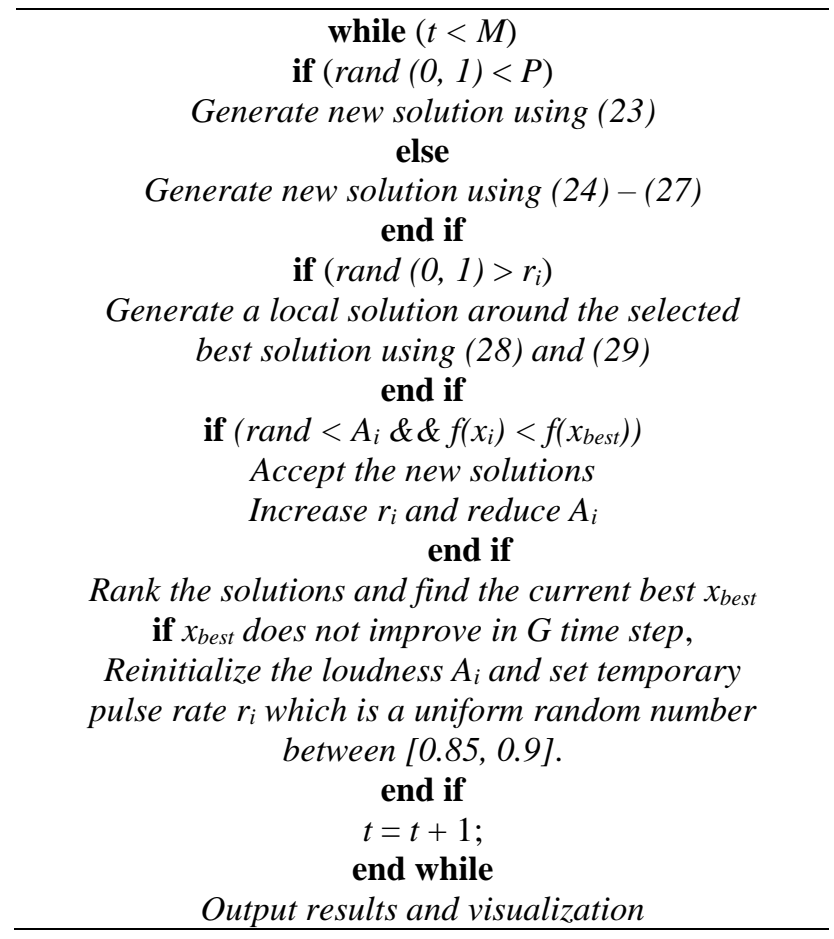

\section{Results and discussion}

\subsection{IEEE 30-bus test system}

The proposed NBA technique has been tested on the standard IEEE 30-bus test system which is shown in Figure 1. The system consists of 41 transmission lines, 6 generating units and 4 tap-changing transformers. The complete system data is given in $[33,34]$. The upper and lower active power generating limits and the fuel cost coefficients of all generators of the standard IEEE 30-bus test system are presented in Table 3 . The voltage magnitude limits are between 0.95 and $1.05 \mathrm{pu}$ for all load buses, while it is between $0.95 \mathrm{and} 1.1 \mathrm{pu}$ for all generator buses. Tap settings of all transformer taps are between 0.9 and 1.1 pu. The total system demand was chosen 283.4 MW. The algorithms were executed in MATLAB R2015a on a PC with 3.07 GHz CPU and 8-GB RAM. The parameters of NBA technique used for simulation are: $\alpha=\gamma=0.9 ; f_{\min }=0 ; f_{\max }$ $=1.5 ; A_{0} \in[0,2] ; r_{0} \in[0,1] ; G=10 ; P \in[0.5,0.9] ; w \in[0.4,0.9] ; C R \in[0.1,0.9] ; \theta \in[0.5,1]$.

The optimal values of the control variables are given in Table 4 . The total fuel cost obtained by the proposed technique is $801.8442 \$ / \mathrm{h}$ and total active power loss is $9.1983 \mathrm{MW}$. Table 5 shows a comparison between the results of fuel cost and power losses obtained from the proposed approach and those reported in the literature. The comparison is performed with the same control variable limits, initial conditions, and other system data. It is clear from the table that the proposed NBA technique outperforms TS, TLBO, BBO, ABC, and GA techniques. Figure 2 shows the convergence characteristic of the NBA for IEEE 30-bus test system.

\subsection{IEEE 57-Bus Test System}

To evaluate the efectiveness and efficiency of the proposed NBA approach in solving larger power system, a standard IEEE 57-bus test system is considered as shown in Figure 3. The standard test system consists of 80 transmission lines, seven gnerators at the buses 1, 2, 3, 6, 8, 9, and 12, and 15 branches under the load tap setting transformer branches. The shunt reactive power sources are considered at buses 18,25 , and 53. The total load demand of the system is 1250.8 MW and 336.4 MVAR. The bus data and the line data are taken from [33, 34]. The minimum and maximum active power generating limits and the fuel cost coefficients of all generators of the standard IEEE 57-bus test system are presented in Table 6. The maximum and minimum values for voltages of all gnerator buses and tap setting transformer control variables are considered to be 1.1-0.9 in p.u. The maximum and minimum values of shunt reactive power sources are 0.0 and 0.3 in p.u. The maximum and minimum values for voltages of all load buses are 1.06 and 0.94 in p.u, respectively. 
Best control variables settings are given in Table 7. The total fuel cost obtained by proposed technique is $41838.2081 \$ / \mathrm{h}$ and total active power loss is $18.6718 \mathrm{MW}$. Table 8 shows a comparison between the results of fuel cost obtained from the proposed approach and those reported in the literature. The comparison is performed with the same control variable limits, initial conditions, and other system data. It is clear from the table that the proposed NBA technique outperforms Shuffled Frog Leaping Algorithm (SFLA) and Grey Wolf Optimizer (GWO) techniques. Figure 4 show the convergence characteristic of the NBA for IEEE 57bus test system.

Table 3. Generator data and fuel cost coefficients.

\begin{tabular}{|c|c|c|c|c|c|}
\hline Bus & $\begin{array}{c}P_{G i}^{\min } \\
(\mathrm{MW})\end{array}$ & $\begin{array}{c}P_{G i}^{\max } \\
(\mathrm{MW})\end{array}$ & $\begin{array}{c}a_{i} \\
(\$ / \mathrm{h})\end{array}$ & $b_{i}(\$ / \mathrm{MWh})$ & $\begin{array}{c}c_{i} \\
\left(\$ / \mathrm{MW}^{2} \mathrm{~h}\right)\end{array}$ \\
\hline 1 & 50 & 200 & 0.00 & 2.00 & 0.00375 \\
\hline 2 & 20 & 80 & 0.00 & 1.75 & 0.01750 \\
\hline 5 & 15 & 50 & 0.00 & 1.00 & 0.06250 \\
\hline 8 & 10 & 35 & 0.00 & 3.25 & 0.00834 \\
\hline 11 & 10 & 30 & 0.00 & 3.00 & 0.02500 \\
\hline 13 & 12 & 40 & 0.00 & 3.00 & 0.02500 \\
\hline
\end{tabular}

Table 4. Optimal values of control variables.

\begin{tabular}{|c|c|c|c|}
\hline \multirow{2}{*}{ Variables } & \multicolumn{2}{|c|}{ Limit } & \multirow{2}{*}{$\begin{array}{c}\text { Result } \\
\text { (Best solution) }\end{array}$} \\
\hline & Lower & Upper & \\
\hline$P_{G 1}(\mathrm{MW})$ & 50 & 200 & 177.1036 \\
\hline$P_{G 2}(\mathrm{MW})$ & 20 & 80 & 48.7199 \\
\hline$P_{G 5}(\mathrm{MW})$ & 15 & 50 & 21.5012 \\
\hline$P_{G 8}(\mathrm{MW})$ & 10 & 35 & 21.8577 \\
\hline$P_{G 11}(\mathrm{MW})$ & 10 & 30 & 12.1606 \\
\hline$P_{G 13}(\mathrm{MW})$ & 12 & 40 & 12.2553 \\
\hline$V_{G 1}(\mathrm{pu})$ & 0.95 & 1.10 & 1.0600 \\
\hline$V_{G 2}(\mathrm{pu})$ & 0.95 & 1.10 & 1.0430 \\
\hline$V_{G 5}(\mathrm{pu})$ & 0.95 & 1.10 & 1.0100 \\
\hline$V_{G 8}(\mathrm{pu})$ & 0.95 & 1.10 & 1.0100 \\
\hline$V_{G 11}(\mathrm{pu})$ & 0.95 & 1.10 & 1.0820 \\
\hline$V_{G 13}(\mathrm{pu})$ & 0.95 & 1.10 & 1.0710 \\
\hline$T_{4-12}(\mathrm{pu})$ & 0.90 & 1.10 & 0.9393 \\
\hline$T_{6-9}(\mathrm{pu})$ & 0.90 & 1.10 & 1.0165 \\
\hline$T_{6-10}(\mathrm{pu})$ & 0.90 & 1.10 & 0.9957 \\
\hline$T_{27-28}(\mathrm{pu})$ & 0.90 & 1.10 & 0.9285 \\
\hline $\begin{array}{c}J(\$ / \mathrm{h}) \\
\text { Ploss }(\mathrm{MW})\end{array}$ & \multicolumn{3}{|c|}{$\begin{array}{c}801.8442 \\
9.1983\end{array}$} \\
\hline
\end{tabular}




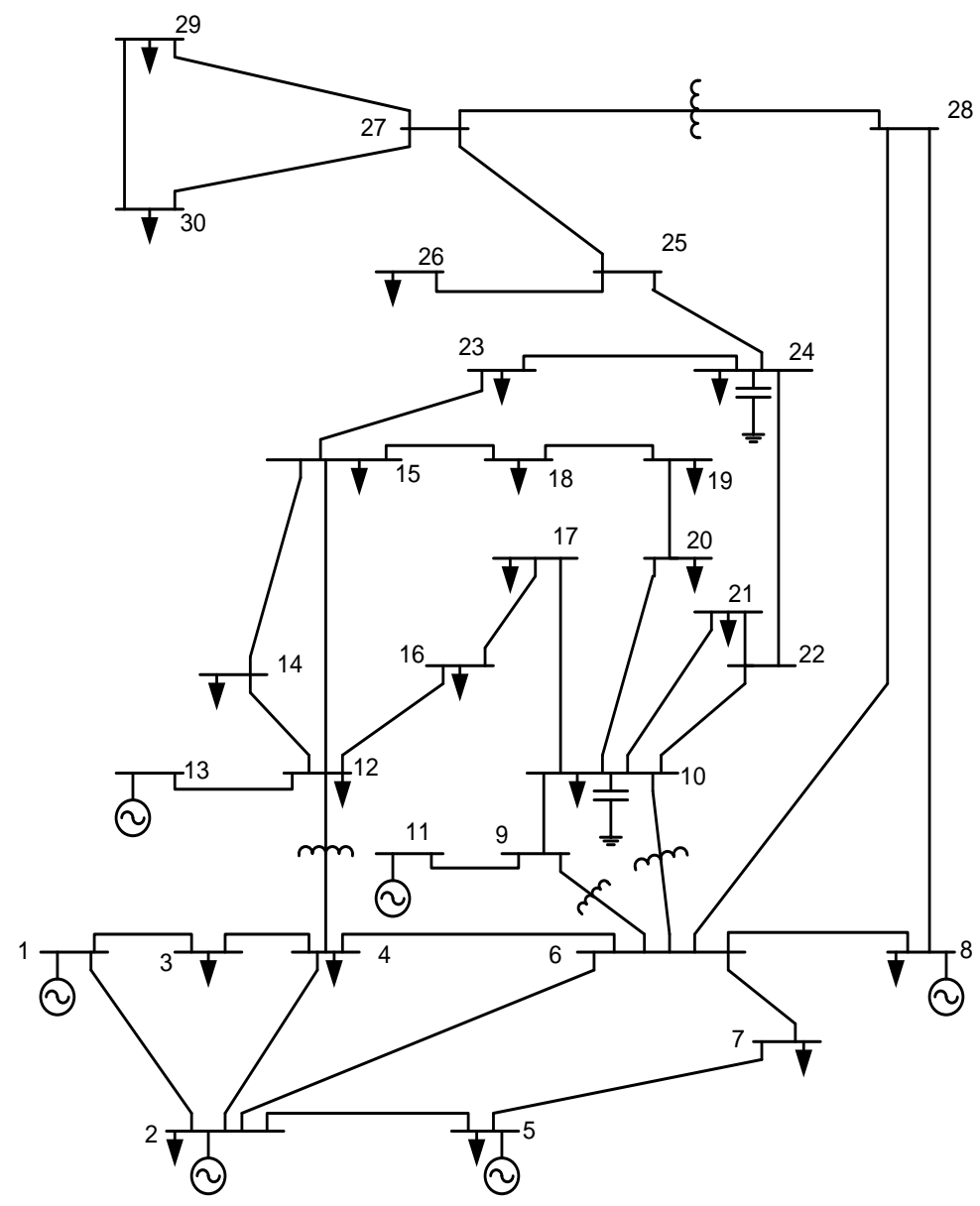

Figure 1. Single line diagram of the IEEE 30-bus test system.

Table 5. Results of minimum fuel cost for the IEEE 30-bus system.

\begin{tabular}{|c|c|c|c|c|c|c|}
\hline Variables & TS [5] & TLBO [19] & BBO [22] & ABC [22] & GA [22] & NBA \\
\hline$P_{G 1}(\mathrm{MW})$ & 176.04 & 176.94 & 171.9231 & 180.5218 & 177.28 & 177.1036 \\
$P_{G 2}(\mathrm{MW})$ & 48.75 & 49.02 & 48.8394 & 48.7845 & 48.817 & 48.7199 \\
$P_{G 5}(\mathrm{MW})$ & 21.56 & 21.53 & 21.4391 & 21.2598 & 21.529 & 21.5012 \\
$P_{G 8}(\mathrm{MW})$ & 22.05 & 21.81 & 21.7629 & 18.6469 & 21.81 & 21.8577 \\
$P_{G 11}(\mathrm{MW})$ & 12.44 & 12.20 & 12.1831 & 11.8145 & 11.325 & 12.1606 \\
$P_{G 13}(\mathrm{MW})$ & 12.00 & 11.41 & 16.5588 & 12.1011 & 12.087 & 12.2553 \\
\hline Fuel cost (\$/h) & 802.29 & 802.45 & 802.717 & 802.1649 & 802.0012 & 801.8442 \\
\hline Power loss (MW) & - & 9.525 & 9.3064 & 9.7286 & 9.4563 & 9.1983 \\
\hline
\end{tabular}

Table 6. Generator data and fuel cost coefficients.

\begin{tabular}{|c|c|c|c|c|c|}
\hline Bus & $\begin{array}{c}P_{G i}^{\min } \\
(\mathrm{MW})\end{array}$ & $\begin{array}{c}P_{G i}^{\max } \\
(\mathrm{MW})\end{array}$ & $\begin{array}{c}a_{i} \\
(\$ / \mathrm{h})\end{array}$ & $b_{i}(\$ / \mathrm{MWh})$ & $\begin{array}{c}c_{i} \\
\left(\$ / \mathrm{MW}^{2} \mathrm{~h}\right)\end{array}$ \\
\hline 1 & 0 & 575.88 & 0.00 & 20 & 0.07758 \\
\hline 2 & 0 & 100 & 0.00 & 40 & 0.01000 \\
\hline 3 & 0 & 140 & 0.00 & 20 & 0.25000 \\
\hline 6 & 0 & 100 & 0.00 & 40 & 0.01000 \\
\hline 8 & 0 & 550 & 0.00 & 20 & 0.022222 \\
\hline 9 & 0 & 100 & 0.00 & 40 & 0.01000 \\
\hline 12 & 0 & 410 & 0.00 & 20 & 0.032258 \\
\hline
\end{tabular}




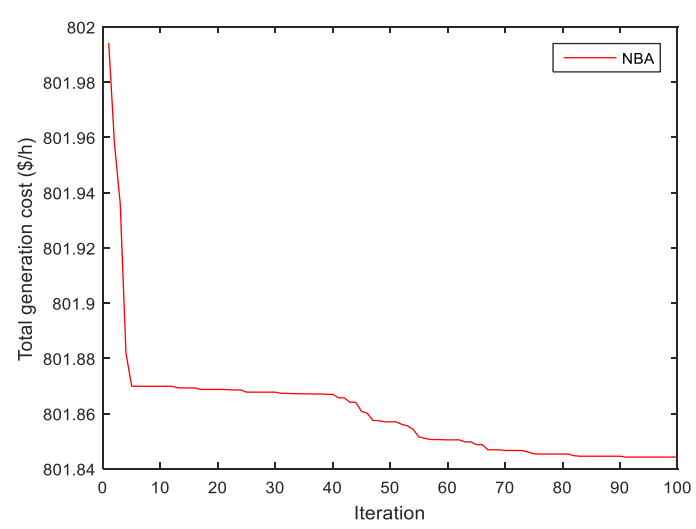

Figure 2. Convergence characteristic of the NBA for IEEE 30-bus test system.

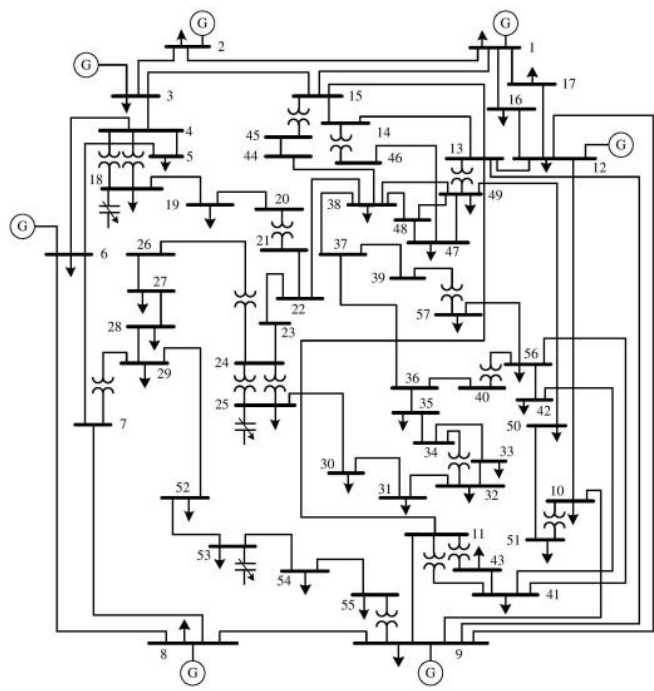

Figure 3. Single line diagram of the IEEE 57-bus test system.

Table 7. Best control variables settings for the IEEE57-bus test system.

\begin{tabular}{|c|c|c|c|}
\hline Control variables & Best result & Control variables & Best result \\
\hline$P_{G 1}(\mathrm{MW})$ & 144.8940 & $T_{24-25}(\mathrm{pu})$ & 0.9062 \\
$P_{G 2}(\mathrm{MW})$ & 85.9112 & $T_{24-25}(\mathrm{pu})$ & 1.0468 \\
$P_{G 3}(\mathrm{MW})$ & 47.4731 & $T_{24-26}(\mathrm{pu})$ & 1.0014 \\
$P_{G 6}(\mathrm{MW})$ & 76.1576 & $T_{7-29}(\mathrm{pu})$ & 0.9619 \\
$P_{G 8}(\mathrm{MW})$ & 457.6745 & $T_{34-32}(\mathrm{pu})$ & 0.9613 \\
$P_{G 9}(\mathrm{MW})$ & 93.2460 & $T_{11-41}(\mathrm{pu})$ & 0.9404 \\
$P_{G 12}(\mathrm{MW})$ & 364.1153 & $T_{11-43}(\mathrm{pu})$ & 0.9773 \\
$V_{G 1}(\mathrm{pu})$ & 1.0400 & $T_{15-45}(\mathrm{pu})$ & 0.9832 \\
$V_{G 2}(\mathrm{pu})$ & 1.0200 & $T_{14-46}(\mathrm{pu})$ & 0.9973 \\
$V_{G 3}(\mathrm{pu})$ & 0.9950 & $T_{10-51}(\mathrm{pu})$ & 0.9485 \\
$V_{G 6}(\mathrm{pu})$ & 1.0000 & $T_{13-49}(\mathrm{pu})$ & 1.0397 \\
$V_{G 8}(\mathrm{pu})$ & 1.0050 & $T_{9-55}(\mathrm{pu})$ & 0.9709 \\
$V_{G 9}(\mathrm{pu})$ & 0.9900 & $T_{40-56}(\mathrm{pu})$ & 0.9680 \\
$V_{G 12}(\mathrm{pu})$ & 1.0150 & $T_{39-57}(\mathrm{pu})$ & 0.9830 \\
$T_{4-18}(\mathrm{pu})$ & 0.9354 & $Q_{\mathrm{c} 18}(\mathrm{pu})$ & 0.1522 \\
$T_{4-18}(\mathrm{pu})$ & 0.9364 & $Q_{\mathrm{c} 25}(\mathrm{pu})$ & 0.1436 \\
$T_{21-20}(\mathrm{pu})$ & 1.0339 & $Q_{\mathrm{c} 53}(\mathrm{pu})$ & 0.1508 \\
\hline \multicolumn{2}{|c|}{$J(\$ / \mathrm{h})=41838.2081 ;$} & Ploss $(\mathrm{MW})=18.6718$ \\
\hline
\end{tabular}




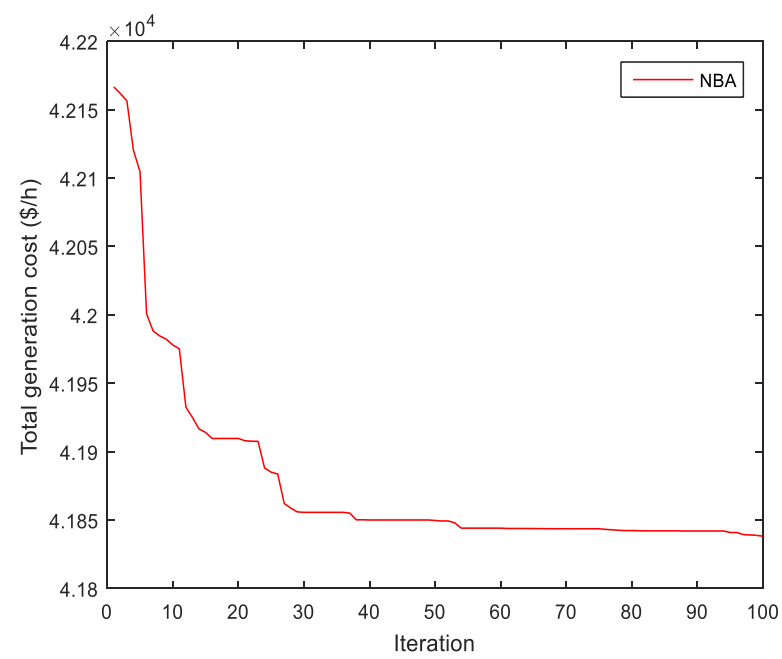

Figure 4. Convergence characteristic of the NBA for IEEE 57-bus test system.

Table 8. Results of minimum fuel cost for the IEEE 57-bus system.

\begin{tabular}{|c|c|c|c|}
\hline Variables & SFLA [35] & GWO [35] & NBA \\
\hline$P_{G 1}(\mathrm{MW})$ & 144.856 & 145.42 & 144.8940 \\
$P_{G 2}(\mathrm{MW})$ & 93.0378 & 95.66 & 85.9112 \\
$P_{G 3}(\mathrm{MW})$ & 45.209 & 45.02 & 47.4731 \\
$P_{G 6}(\mathrm{MW})$ & 68.2624 & 67.57 & 76.1576 \\
$P_{G 8}(\mathrm{MW})$ & 457.0264 & 454.28 & 457.6745 \\
$P_{G 9}(\mathrm{MW})$ & 95.8565 & 94.11 & 93.2460 \\
$P_{G 12}(\mathrm{MW})$ & 365.9573 & 367.95 & 364.1153 \\
\hline Fuel cost $(\$ / \mathrm{h})$ & 41872.9 & 41873.188 & 41838.2081 \\
\hline
\end{tabular}

\section{Conclusion}

In this paper, a novel bat algorithm (NBA) technique has been applied to solve the OPF problem in the power system. The proposed technique has provided the global solution in the standard IEEE 30-bus and IEEE 57-bus test systems, and it has produced results which are better than the previous studies reported in the literature. The obtained results indicate that the proposed technique can be effectively used to solve the optimal power flow problem.

\section{References}

[1] Dommel, H.W., Tinny, W. F.: Optimal power flow solutions, IEEE Transactions on Power Apparatus Systems, PAS-87 (1968), 5, 1866-1876.

[2] Momoh, J. A., Adapa, R., El-Hawary, M. E.: A review of selected optimal power flow literature to 1993. I. nonlinear and quadratic programming approaches, IEEE Transactions on Power Systems, 14(1999), 1, 96-104.

[3] Momoh, J. A., El-Hawary, M. E., Adapa, R.: A review of selected optimal power flow literature to 1993. II. Newton, linear programming and interior point methods, IEEE Transactions on Power Systems, 14 (1999), 1, 105-111.

[4] Huneault, M., Galiana, F. D.: A survey of the optimal power flow literature, IEEE Transactions on Power Systems, 6 (1991), 2, 762-770.

[5] Abido, M. A.: Optimal power flow using tabu search algorithm, Electric Power Components and Systems, 30 (2002), 5, 469-483.

[6] Lai, L. L., Ma, J. T., Yokoyama, R., Zhao, M.: Improved genetic algorithms for optimal power flow under normal and contingent operation states, International Journal of Electric Power and Energy Systems, 19 (1997), 5, 287-292.

[7] Bakirtzis, A. G., Biskas, P. N., Zoumas, C. E., Pehdis, V.: Optimal power flow by enhanced genetic algorithm, IEEE Transactions on Power Systems, 17 (2002), 2, 229-236. 
[8] Deveraj, D., Yegnanarayana, B.: Genetic algorithm based optimal power flow for security enhancement, IEE Proc. Gener. Transm. Distrib., 152 (2005), 6, 899-905.

[9] Jayabarathi, T., Sadasivam, G.: Evolutionary programming-based economic dispatch for units with multiple fuel options, European Transactions on Electrical Power, 10 (2000), 3, 167-170.

[10] Luong Le Dinh, Dieu Vo Ngoc, Pandian Vasant: Artificial bee colony algorithm for solving optimal power flow problem, The Scientific Word Journal, 2013 (2013), pp. 1-9.

[11] Christy, A. A., Ajay, D. P., Vimal Raj, Anbumozi, R.: A new optimization algorithm for solving optimal power flow, International Journal of Advanced Research in Electrical, Electronics and Instrumentation Engineering, 3 (2014), 2, 41-52.

[12] Mouassa, S., Bouktir, T.: Artificial bee colony algorithm for solving OPF problem considering the valve point effect, International Journal of Computer Applications, 112 (2015), 1, 45-53.

[13] Sayah, S., Zehar, K.: Modified differential evolution algorithm for optimal power flow with nonsmooth cost functions, Energy Conversion and Management, 49 (2008), 3036-3042.

[14] Abou El Ela, A. A., Abido, M. A., Spea, S. R.: Optimal power flow using differential evolution algorithm, Springer-Verlag, Electrical Engineering, 8 (2009), 69-78.

[15] Abou El Ela, A. A., Abido, M. A.: Optimal power flow using differential evolution algorithm, Electric Power Systems Research, 80 (2010), 7, 878-885.

[16] Vaisakh, K., Srinivas, L. R.: Differential evolution approach for optimal power flow solution, Journal of Theoretical and Applied Information Technology, (2008), 261-268.

[17] Nayak, M. R., Nayak, C. K., Rout, P. K.: Application of multi-objective teaching learning-based optimization algorithm to optimal power flow problem, in Procedia Technology, 6 (2012), 255-264.

[18] Bouchekara, H.R.E.H., Abido, M. A., Boucherma, M.: Optimal power flow using teaching-learningbased optimization technique, Electric Power Systems Research, 114 (2014), 49-59.

[19] Gnanambal, K., Jayavelumani, K. R., Juriya Banu, H.: Optimal power flow using hybrid teaching learning-based optimization algorithm, in International Conference on Innovations in Engineering and Technology (ICIET-2016), 2016, 237-243.

[20] Roy, P. K., Ghoshal, S. P., Thakur, S. S.: Biogeography based optimization for multi-constraint optimal power flow with emission and non-smooth cost function, Expert System with Applications, 37 (2010), 8221-8228.

[21] Roy, P. K., Ghoshal, S. P., Thakur, S. S.: Biogeography based optimization approach for optimal power flow problem considering valve loading effects, International Journal of Recent Trends in Engineering and Technology, 3 (2010), 3, 177-181.

[22] Herbadji, O., Slimani, L., Bouktir, T.: Biogeography based optimization approach for solving optimal power flow problem, International Journal of Hybrid Information Technology, 6 (2013), 5, 183-196.

[23] Abido, M. A.: Optimal power flow using particle swarm optimization, Electrical Power and Energy Systems, 24 (2002), 563-571.

[24] Hazra, J., Sinha, A. K.: A multi-objective optimal power flow using particle swarm optimization, European Transactions on Electrical Power, 21 (2011), 1, 1028-1045.

[25] Al-Bahrani, L., Dumbrava, V.: Optimal power flow based on particle swarm optimization, U.P.B. Sci. Bull., Series C, 78 (2016), 3, 253-264.

[26] Duman, S., Güvenç, U., Sönmez, Y., Yörükeren, N.: Optimal power flow using gravitational search algorithm, Energy Conversion and Management, 59 (2012), 86-95.

[27] Yang, X. S.: A new metaheuristic bat-inspired algorithm, in: Nature Inspired Cooperative Strategies for Optimization (NISCO 2010) (Eds. Cruz, C.; Gonz'alez, J. R.; Pelta, D. A.; Terrazas, G), Studies in Computational Intelligence, 284 (2010), Springer Berlin, 65-74.

[28] Yang, X. S.: Bat algorithm for multiobjective optimization, Int. J. Bio-Inspired Computation, 3 (2011), $5,267-274$.

[29] Yang, X. S.: Bat algorithm: literature review and applications, Int. J. Bio-Inspired Computation, 5 (2013), 3, 141-149.

[30] Meng, X. B., Gao, X. Z., Liu, Y., Zhang, H.: A novel bat algorithm with habitat selection and doppler effect in echoes for optimization, Expert Systems with Applications, 42 (2015), 6350-6364.

[31] Gautham, S., Rajamohan, J.: Economic load dispatch using novel bat algorithm, IEEE 1st International Conference on Power Electronics, Intelligent Control and Energy Systems (ICPEICES), Delhi, India, 4-6 July 2016. 
[32] Hardiansyah: Non-convex economic load dispatch problems using novel bat algorithm, Journal of Theoretical and Applied Information Technology (JATIT), 96 (2018), 8, 2361-2369.

[33] Alsac, O., Stott, B.: Optimal load flow with steady-state security, IEEE Transactions on Power Apparatus Systems, PAS-93 (1974), 3, 745-751.

[34] Lee, K. Y., Park, Y. M., Ortiz, J. L.: A united approach to optimal real and reactive power dispatch, IEEE Transactions on Power Apparatus Systems, PAS-104 (1985), 5, 1147-1153.

[35] Khamees, A. K., El-Rafei, A., Badra, N. M., Abdelaziz, A. Y.: Solution of optimal power flow using evolutionary-based algorithms, International Journal of Engineering, Science and Technology, 9 (2017), 1, 55-68. 